Voix et Images

\title{
Variations de la critique aquinienne : de la pragmatique à la psychanalyse lacanienne en passant par Bakhtine
}

\section{Jacques Pelletier}

Volume 18, numéro 3 (54), printemps 1993

Littérature, folie, altérité

URI : https://id.erudit.org/iderudit/201054ar

DOI : https://doi.org/10.7202/201054ar

Aller au sommaire du numéro

Éditeur(s)

Université du Québec à Montréal

ISSN

0318-9201 (imprimé)

1705-933X (numérique)

Découvrir la revue

\section{Citer cet article}

Pelletier, J. (1993). Variations de la critique aquinienne : de la pragmatique à la psychanalyse lacanienne en passant par Bakhtine. Voix et Images, 18(3),

597-605. https://doi.org/10.7202/201054ar d'utilisation que vous pouvez consulter en ligne. 


\title{
Variations de la critique aquinienne: de la pragmatique à la psychanalyse lacanienne en passant par Bakhtine
}

\author{
Jacques Pelletier, Université du Québec à Montréal
}

Dans l'ensemble de la production romanesque québécoise, et ce depuis les origines, il semble bien que l'œuvre d'Aquin soit celle qui ait retenu jusqu'ici l'attention la plus soutenue des critiques et spécialistes en tous genres de la discipline.

Du vivant de l'auteur déjà cette œuvre faisait l'objet de nombreuses études relevant pour l'essentiel de deux traditions majeures: d'une part l'analyse structurale, formelle du texte à l'époque de la sémiotique triomphante au début des années 1970 - courant dont l'ouvrage de Françoise Iqbal ${ }^{1}$, paru en 1978, constitue une bonne illustration -; d'autre part la "lecture nationaliste", inaugurée brillamment par Patricia Smart dans son. ouvrage fondateur ${ }^{2}$ en 1973 et reprise avec plus ou moins de bonheur par la suite par un Gilles de la Fontaine ${ }^{3}$, entre autres, en 1978.

Au tournant des années 1980 on voit apparaitre de nouvelles analyses et interprétations: une réflexion d'ordre philosophique et esthétique sur la signification de cette entreprise d'écriture dans les travaux de René Lapierre ${ }^{4}$; une relecture dans la perspective religiologique d'une quête du sacré par Pierre-Yves Mocquais's en 1985; une prise en compte du contexte biographique dans le travail d'enquête de Françoise Iqbal ${ }^{6}$, en 1987 , et plus récemment de Guylaine Massoutre ${ }^{7}$. Et voici que s'ajoutent encore de nouvelles approches, des éclairages souvent inédits dans des essais parus depuis deux ans, relevant de l'intertextualité, domaine de recherche très fréquenté depuis quelques années, de la pragmatique, courant également récent en études littéraires, de l'histoire revue et corrigée dans une optique bakhtinienne, 
et enfin de la psychanalyse lacanienne. Bref cette œuvre n'échappe, si j'ose dire, à aucun courant important parmi les discours tenus aujourd'hui sur la littérature. Sa connaissance en sort-elle, pour autant, renouvelée, approfondie, enrichie? Comment et jusqu'à quel point?

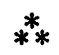

André Lamontagne, dans Les Mots des autres ${ }^{8}$, se réclame de ce qu'il appelle un "structuralisme ouvert * (p. 9), c'est-à-dire reposant sur les acquis méthodologiques de la première vague structuraliste mais en refusant la raideur et le sectarisme. Il préconise et pratique une approche en douceur de l'intertextualité qui lui parait une caractéristique centrale des œuvres modernes et postmodernes, notamment celle d'Aquin.

Son ouvrage, comme il est d'usage dans toute bonne thèse qui se respecte, s'ouvre sur un rappel historique des théories de l'intertextualité qui en fait défiler les principaux représentants, de Bakhtine à Genette, en passant, bien sûr, par Kristeva. Rappel utile, bien fait, qui retient l'essentiel et qui dégage nettement les orientations personnelles de l'auteur. Suit une première évocation, sur le mode descriptif, des diverses formes que prend cette pratique chez Aquin: citations littérales ou plus ou moins déformées, résumés-synthèses, allusions, plagiats explicites ou voilés, transpositions en tous genres, etc. Cette description fait l'objet d'un traitement minutieux, très élaboré, souvent technique et, disons-le, parfois inutilement détaillé car non relié à une analyse d'ensemble de la fonction et de la signification des "emprunts" d'Aquin.

Le travail de Lamontagne devient vraiment éclairant et intéressant lorsqu'il se livre à cette mise en rapport dans la deuxième partie de l'ouvrage où il examine la nature et le statut des principaux intertextes dans le roman. Dans le chapitre sur Prochain Épisode, il montre très bien comment la référence à Balzac et à Simenon, notamment, fait sens dans le cadre d'un récit traversé par la double thématique de l'impuissance (historique et littéraire) et de l'originalité (d'un projet social et d'écriture). Dans le chapitre sur Trou de mémoire, il met en lumière la fonction compensatoire d'une érudition débordante, expansive érigée sur le constat d'un vide aussi bien existentiel que politique et culturel. Solution trompeuse, leurre révélateur, selon lui, d'une entreprise qui ne parvient pas à produire une véritable écriture, à se déployer de manière autonome et créatrice. Cette tendance s'accentue, 
selon Lamontagne, dans L'Antiphonaire, s'aggrave pour ainsi dire dans un * plagiat massif " qui confine l'œuvre à l'autotoreflexivité, à l'autocommentaire, à la prison dans l'x espace littéraire " dont Aquin ne pourra plus s'échapper par la suite, Neige noire apparaissant comme l'ultime manifestation d'une *impuissance littéraire" (p. 217) indépassable, insurmontable. Dans cette perspective, conclut-il, "le travail intertextuel, et principalement l'interdiscursivité et l'hypertextualité, n'exerce pas son pouvoir différenciateur, mais contribue plutôt à la faillite de l'écriture* (p. 224).

Cette conclusion, bien entendu, est discutable et on peut légitimement, pour de fort bonnes raisons, ne pas la faire sienne. Reste qu'elle intervient au terme d'une analyse tout à fait convaincante et allègrement menée du rapport à l'Autre (écrivains, savants, philosophes, etc.) dans l'œuvre d'Aquin et qu'elle éclaire une dimension essentielle, un fondement de sa complexité et de sa richesse.

L'ouvrage de Lamontagne vaut aussi pour la réflexion qu'il contient sur la postmodernité d'Aquin. Question difficile, pleine d'embûches - qu'est-ce que le moderne et le postmoderne? - qu'il aborde au moyen d'une étude comparative des œuvres de Borges et d'Aquin. L'intertextualité, dans la conception qu'il retient du postmoderne, en est un élément constitutif, sans doute le plus important de cette entreprise dont la définition demeure - - encere - flottante. L'apport spécifique d'Aquin réside dans l'importance qu'il accorde à l'action dynamique, créatrice du lecteur par qui l'écriture trouve son véritable avènement.

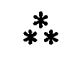

Anthony Wall ${ }^{9}$ inscrit pour sa part son projet dans la lignée de Patricia Smart, réactualisée en quelque sorte à la lumière de Bakhtine. L'objectif est de relire Aquin dans une perspective politique renouvelée, au-delà des références explicites mises en relief naguère par De la Fontaine, Smart et les critiques nationalistes.

Il s'agit donc de revenir à une analyse des enjeux culturels et sociaux de cette ouvre que les études formelles ne prennent pas suffisamment en considération à partir toutefois d'une nouvelle approche, mettant l'accent sur l'imbrication fort étroite, la complémentarité nécessaire liant chez Aquin le travail de la métaphorisation à celui de la référentialisation. L'hypothèse retenue - objet de la démonstration de Wall - est que la métaphore est ici le moyen obligé et 
l'instrument privilégié de la référence. Si bien que ce qui servirait de toile de fond et par suite de principe d'intelligibilité de l'œuvre, ce serait ce que l'auteur appelle le "référent abstrait " que constituerait le Québec souhaité par Aquin, pays indépendant enfin débarrassé de l'oppression coloniale.

Ce "référent abstrait", par ailleurs, ne serait pas représenté explicitement - par définition - dans les romans mais se laisserait lire et appréhender à travers une élaboration métaphorique sophistiquée, le festonnage scripturaire servant à la fois d'écran et de révélateur, à condition bien sûr d'en posséder le code.

C'est ainsi que dans Prochain Épisode le Québec se profile derrière la Suisse, que la mise en scène de celle-ci n'a de sens que dans son rapport à la question nationale. De même, sur un autre plan, l'incohérence formelle qui caractérise Trou de mémoire ne peut être vraiment expliquée qu'en tant qu'équivalent littéraire de l'incohérence et de l'équivoque de la situation nationale québécoise et il n'est pas besoin pour démontrer cela de rabattre l'analyse des personnages et de leurs rapports sur le contexte réel de la condition d'exploitation culturelle et sociale des Québécois comme l'ont fait certains critiques. Dans L'Antiphonaire, le "mouvement chronotopique", l'aller-retour incessant du Québec contemporain au $x^{e} I^{e}$ siècle européen participe de la même volonté de rendre compte d'un ici et maintenant d'une condition québécoise marquée par l'oubli, l'absence et la dépossession. Enfin, dans Neige noire, comme l'écrit Aquin lui-même, le Québec est "en creux", dissimulé sous une représentation, une stylisation apparemment artifi-. cielle qui, pour Lamontagne, explique l'insuffisance de la littérature alors qu'elle signifie, pour Wall, une "expérience de la vérité esthétique, expérience de la vérité vitale» ( $p$. 207). On voit ici comment le point de vue adopté peut déterminer une évaluation, négative chez Lamontagne qui fait primer l'écriture envisagée formellement, positive chez Wall qui la prend en compte dans son rapport à l'Histoire.

C'est que pour Wall la littérature est un acte, une praxis qui doit être inspirée par "un but émotif de travail sur le lecteur" (p. 209). C'est en tous les cas l'intention, la visée profonde qu'il retrace chez Aquin et qu'il semble partager: "Le politique, écrit-il, réside dans la façon dont la vérité esthétique de la métaphorisation peut transformer la vision du lecteur devant son vécu "(p. 212). C'est dans la mesure où l'œuvre d'Aquin contribue à opérer cette métamorphose, cette transfiguration du regard - et par suite des pratiques vécues - qu'elle exerce un effet réel sur la situation concrète du Québec, qu'elle suscite un passage de la métaphore à la référence, s'offrant comme un opé- 
rateur dynamique de cette culture et de cette société, comme un agent capital de sa transformation en véritable "cité libre".

\section{***}

L'étude de Marilyn Rándall ${ }^{10}$ se situe à sa manière dans le prolongement des travaux de Lamontagne et de Wall. Comme celui-ci elle consacre l'essentiel de ses analyses aux rapports. entre l'identité nationale et le travail formel désarticulé qu'exprime l'œuvre d'Aquin. Comme Lamontagne elle s'intéresse à la mise en forme de l'intertextualité qui, dans Trou de mémoire, se présente comme une véritable "poétique du plagiat". Elle se démarque toutefois de Wall dans la mesure où elle envisage la "référence " d'abord et surtout au niveau du contexte discursif et de Lamontagne en ce qu'elle limite son analyse de l'intertextualité à un seul roman, Trou de mémoire.

Son approche, qui se réclame de la pragmatique, veut éviter et dépasser le double écueil de la sémiotique littéraire, axée sur la description formelle du texte, et de l'herméneutique centrée sur son interprétation. Elle considère l'œuvre comme le maillon fort d'une situation communicative impliquant le producteur du texte, le lecteur et les conditions de production et de réception dans lesquelles ils interviennent. Dans cette perspective la notion de contexte est tout à fait capitale, bien que Randall ait tendance, sur le plan théorique, à la limiter un peu étroitement au plan discursif.

Dans la pratique toutefois elle prend aussi en considération le contexte culturel et social par quoi on peut expliquer dans une large mesure l'entreprise d'Aquin. C'est ainsi par exemple qu'elle lit le "trou de mémoire", métaphore principale du roman qui ne prend sa signification que rapportée à l'interprétation de la situation coloniale opérée par Aquin. Elle rend compte de même de la curieuse forme de "roman policier" empruntée par le récit, marquée par les contradictions, les incohérences, les impasses, désarticulation qui se manifeste comme l'expression d'une contestation non pas seulement formelle, "littéraire", d'un genre mais sa subversion pour des raisons "politiques": "La rupture de la cohérence, prônée par Aquin et les Parti pristes, écrit-elle, est une stratégie par laquelle ils signalent leur nonappartenance aux contextes littéraire et social ambiants". (p. 90). Randall rejoint par là l'analyse de Wall, insistant sur le caractère nécessaire, contraignant, de la "trame historique" dans la structuration du roman, dans sa mise en forme textuelle. L'architectonique de 
l'œuvre trouve de la sorte ses fondements dans le contexte discursif et social où intervient Aquin durant les années 1960.

Sur le plan littéraire, son engagement se traduit cependant, et à première vue paradoxalement, par une recherche formelle qui paraît étrangère à ses choix culturels et politiques. Et pourtant c'est sur ce plan qu'elle trouve son ancrage et sa signification profonde. Dans Trou de mémoire, c'est la forme de la citation explicite, du plagiat délibéré qu'emprunte le romancier, en faisant un principe d'élaboration, de construction de son texte en recourant à des manœuvres aussi astucieuses que déconcertantes pour un lecteur qui se retrouve tiraillé entre la fascination et l'agacement devant ce monstrueux étalage d'érudition échevelée et d'incohérence narrative:

Texte constitué de fragments volés et appropriés, souvent sans la moindre ré-écriture, la facture de Trou de mémoire réalise ce que le titre annonce: l'oubli de soi a pour conséquence la dépossession de la parole et l'invasion du malade par la parole de l'autre. Le sujet amnésique est aussi frappé d'aphasie. Ainsi le plagiat et la répétition rejoignent la thématique de la décolonisation... (p. 217).

Cette conclusion est précédée de longues analyses, très fines et minutieuses, des formes concrètes que prend le travail d'encodage d'Aquin dans le roman - à titre d'exemple je renvoie à la remarquable étude de l'élaboration effectuée par le romancier à partir de l'image de la "chambre obscure. (p. 193-194): c'est un modèle dans le genre - et de la manière dont, tout en se distanciant de son objet, en le voilant, il en manifeste par cela même l'irrécurable prégnance. En cela son "anti-littérature", pour reprendre l'expression de Randall, ne signifierait pas nécessairement une évasion dans "l'espace littéraire" comme le prétend Lamontagne, mais se présenterait plutôt comme la façon particulière par laquelle Aquin a choisi de se situer à la fois dans le champ littéraire et dans l'Histoire de la société québécoise.

\section{***}

"À roman baroque, théorisation baroque", ce n'est pas moi qui décris ainsi de manière polémique le projet de Robert Richard ${ }^{11}$ mais bien l'auteur lui-même qui ouvre son ouvrage par cette caractérisation on ne peut plus appropriée à un propos qui relève pour l'essentiel de la psychanalyse lacanienne, courant controversé de la discipline, discuté et discutable particulièrement lorsqu'il s'applique, avec toute la liberté qu'il s'autorise et pour ainsi dire sans contrôle, au texte littéraire. 
Richard, qui ne manque pas de prétention, affirme dans l'Avantpropos de son ouvrage qu'il était temps de * dire du neuf " sur Aquin à la lumière de ce qu'il appelle la mutation postmoderne, d'en prendre donc une nouvelle mesure et de le replacer dans le contexte de la transformation de la culture occidentale à l'époque moderne dont il aurait été un acteur particulièrement dynamique. Plus précisément, c'est à travers le "code romanesque" qui sous-tend son œuvre qu'Aquin aurait mené à terme *l'ultime destin du discours ou du récit occidental, à savoir la logique de sa fiction. (p. 10).

C'est ce code, véritable structure porteuse de l'œuvre, qu'il s'agira de retracer avec le "maximum de clarté. (p. 9) et de limpidité, précautions rhétoriques que Richard sent le besoin d'énoncer au seuil de son ouvrage, question de rassurer ceux et celles qui n'auraient pas "l'oreille lacanienne et qui, du coup, pourraient se retrouver désemparé-es face à la formulation souvent déroutantes de ses analyses. Ce fameux code, ce n'est ni le «signifié ou [le] sens ultime* (p. 14) du texte, ni son "explication", ni encore une "question purement formelle* (p. 14), mais bien plutôt une clé "dont le dentelé serait continuellement en train de changer de forme, et donc une clé en mesure d'ouvrir toutes les portes et aucune. (p. 18). Áu cas où le lecteur non instruit par *l'écoute lacanienne " - n'aurait toujours pas compris ce qu'est au juste cette clé universelle conduisant partout et nulle part, il pourra toujours se reporter à cette autre définition encore plus éclairante:

C'est dire que le code aquinien n'est ni de l'ordre du signifié (ineffable ou méta) ni de l'ordre du signifiant (rêverie ou tromperie). Il serait plutôt de l'ordre du non-signifié / hors-signifiant et donc de l'ordre du réel lacanien comme condition du signifié/signifiant . (p. 27).

C'est si transparent en effet que seul un imbécile ne saurait pas comprendre...

Ainsi armé de ces précisions, le lecteur peut enfin accéder à la révélation, au secret; ce qui soutient l'œuvre d'Aquin, ce qui l'informe de manière implicite mais décisive, c'est ce que Richard appelle "l'inceste généralisé ", un inceste dont on ne retrace des marques explicites que dans de rares passages des romans mais qui constitue néanmoins leur "trame souterraine" d'autant plus évidente, dans cette curieuse logique, qu'elle est absente, l'absence étant ici la garantie en quelque sorte d'une présence.

Cet * inceste généralisé », envahissant, proliférant, traversant l'œuvre de part en part, l'auteur en voit des indices - car il a beau être "absent ", il faut bien qu'il se manifeste quelque part! - d'une part 
dans "l'effondrement du langage * (p. 54) et, d'autre part, dans sa "surstructuration", les deux pratiques éditoriales contradictoires s'offrant comme masques, voiles de l'inceste qu'il faut absolument cacher car littéralement inavouable, comme l'on sait. Par ailleurs, cet inceste dissimulé, refoulé, Richard s'acharne étrangement à en débusquer les diverses et nombreuses occurrences dans les moindres replis du texte, dans les plus minces indices, dans l'espoir - à mon avis, vain - de faire coller son interprétation au "réel du texte qui résiste - et pour cause, : l'inceste généralisé * existant ici d'abord, sinon exclusivement, dans la tête du lecteur/analyste.

Cet * inceste généralisé * est en outre redoublé par rien de moins qu'un *lesbianisme généralisé *, les personnages masculins d'Aquin se révélant d'une certaine manière des "femmes", si bien que l'hétérosexualité ne serait ici qu'une forme renversée de lesbianisme (ou l'inverse, comme on voudra). Là aussi, l'auteur multiplie les "preuves * aussi convaincantes les unes que les autres à l'appui de sa démonstration qui ne fait sens qu'à condition de lire les romans "à la verticale ", position dans laquelle le lecteur se trouve en état de coït amoureux avec le texte, lisant par exemple - je cite! - "droit dans le sexe de K. (p. 56) ou de "Christine (le sexe du Christ). (p. 86)... Comment mieux exprimer la nature fantasmatique, littéralement délirante, de la lecture qui nous est ici proposée, fondée essentiellement sur des projections, de pures constructions d'un imaginaire emballé, surexcité, en proie à une dérive théorique sans objet, sans rapport réel à l'œuvre qui n'existe plus que comme pré-texte, dans tous les sens du terme?

Cette analyse, qu'il me paraîtrait oiseux de rappeler et de discuter dans le détail, s'inscrit en outre dans le cadre d'une réflexion de portée plus générale à laquelle sont convoqués, entre autres, Hegel, Heidegger, Nietzsche et Kant, l'œuvre d'Aquin n'étant rien de moins qu'x assimilable au projet kantien de la raison pratique. (p. 48), sans compter que ses intuitions rejoignent "les élaborations de la psychanalyse lacanienne" (p. 48)! Autant de propositions "songées", comme dirait un jeune d'aujourd'hui, qui laissent pour le moins songeur...

Bref, à l'exception de quelques pages du dernier chapitre qui essaient d'évaluer quel pourrait être l'apport spécifique d'Aquin à la postmodernité telle que pensée par Scarpetta et raffinée par Richard, on cherche en vain quel éclairage vraiment nouveau cet ouvrage apporte à la connaissance de l'œuvre. À force de vouloir trop faire signifier, plus rien ne signifie vraiment, si bien que l'hypersophistication conduit dans le cas qui nous concerne, par un curieux effet de retour, 
à la banalisation la plus extrême. Débarrassé de ses oripeaux lacaniens, le roi est nu.

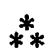

Autant d'études, autant d'analyses et d'interprétations qu'autorise une œuvre polyphonique. et fortement pluridimensionnelle. Autant d'éclairages qui présentent chacun un intérêt spécifique et des' limites dans la mesure où ils ne parviennent pas - mais est-ce possible? - à fournir une représentation d'ensemble, globalisante et totalisante de la production d'Aquin, qui en dégagerait la visée et le sens - ultimes.

Cela dit, il reste qu'on a affaire dans les ouvrages de Lamontagne, Randall et de Wall plus spécifiquement, à certaines percées particulièrement intéressantes sur des questions majeures de la pratique d'Aquin, notamment son rapport à l'autre, complice et rival dans l'écriture, et à un réel qui, pour n'être pas que québécois, trouve d'abord dans le contexte national ses racines et ses fondements. Lues en complémentarité avec les éditions critiques en cours, ces études apportent une contribution significative à la compréhension des enjeux littéraires, culturels et sociaux de cette œuvre majeure du corpus québécois contemporain.

1. Françoise Iqbal, Hubert Aquin romancier, Québec, Presșes de l'Université Laval, coll. - Vie des lettres québécoises , 1978.

2. Patricia Smart, Hubert Aquin, agent double, Montréal, Les Presses de l'Université de Montréal, 1973.

3. Gilles de la Fontaine, Hubert Aquin et le Québec, Montréal, Parti Pris, coll. "Frères chasseurs : 1978.

4. René Lapierre, Les Masques du récit, Montréal, Hurtubise HMH, coll. "Littérature., 1980, et L'Imaginaire captif, Montréal, Quinze, coll. a Prose exacte :, 1982.

5. Pierre-Yves Mocquais, Hubert Aquin ou la Quête interrompue, Montréal, Le Cercle du livre de France, 1985.

6. Françoise Iqbal, Desafinado, Montréal, VLB éditeur, 1987.

7. Guylaine Massoutre, Itinéraires d'Hubert Aquin Montréal, Leméac, coll. «Bibliothèque québécoise., 1992.

8. André Lamontagne, Les Mots des autres. La poétique intertextuelle des ceuvres romanesques de Hubert Aquin, Québec, Les Presses de l'Université Laval, coll. -Vie des lettres québécoises., 1992.

9. Anthóny Wall, Hubert Aquin, entre référence et métaphore, Candiac, Les Éditions Balzac, coll. : L'Univers des discours -, 1991.

10. Marilyn Randall, Le Contexte littéraire: lecture pragmatique de Hubert Aquin et de Réjean Ducharme, Longueuil, Le Préambule, coll. aL'Univers des discours •, 1991.

11. Robert Richard, Le Corps logique de la fiction. Le code romanesque cbez Hubert Aquin, Montréal, l'Hexagone, coll. «Essais littéraire s*, 1990. 